\title{
Spindle Cell Hemangioma and Decorin Expression*
}

\author{
Daiki Rokunohe, Hitoshi Takeda, Takahide Kaneko, Takayuki Aizu, Eijiro Akasaka, \\ Yasushi Matsuzaki, Hajime Nakano, Daisuke Sawamura ${ }^{\#}$
}

Department of Dermatology, Graduate School of Medicine, Hirosaki University, Hirosaki, Japan.

Email: "smartdai@cc.hirosaki-u.ac.jp

Received December $7^{\text {th }}, 2011$; revised December $26^{\text {th }}, 2011$; accepted January $5^{\text {th }}, 2012$

\begin{abstract}
Spindle cell hemangioma (SCH) is a rare benign vascular tumor and we report 2 cases of SCH who are 50-year-old man and 16-year-old girl. A growing amount of evidence indicates that multifunctional ECM molecule decorin regulate endothelial cell-matrix interactions during angiogenesis. Furthermore, a current publication shows that no detectable decorin expression was found in the stromas of tumor nests in Kaposi's sarcoma or angiosarcoma groups while the expression was clearly observed in the connective tissue stromas within benign vascular tumors. SCH exhibits spindle cell proliferation and resembles Kaposi's sarcoma. Therefore, this study examined decorin expression in the tissues of our $\mathrm{SCH}$ cases by immunohistochemical analysis. The results demonstrated that immunoreactivity of decorin was clearly detectable in the connective tissue stroma within tumor nests. SCH was first perceived as sarcoma because of spindle cell proliferation, but this proliferation was subsequently found to be reactive. The presence of decorin expression in our cases also indicates that $\mathrm{SCH}$ have benign properties. Moreover, this study further increases the reliability of examination of decorin expression for differentiation between benign and malignant vascular tumors.
\end{abstract}

Keywords: Proteoglycan; Skin; Endothelium; Malignant; Matrix

\section{Introduction}

Spindle cell hemangioma $(\mathrm{SCH})$ is rare subcutaneous growth presumably derived from malformed vasculature that was first described by Weiss and Enzinger in 1986. $\mathrm{SCH}$ is known as hemangioendothelioma which affects a wide age range and mostly occurs at distal extremities. $\mathrm{SCH}$ was initially believed to be a low-grade angiosarcoma, since proliferation of Kaposi's sarcoma-like spindle cells was detected histologically [1]. However, several studies have revealed that the lesion is not a neoplasm possessing metastatic potential, but a reactive cellular proliferation [2]. Recently, the proteoglycan decorin was demonstrated to play a key role in angiogenesis in normal and tumor-associated endothelium [3-5]. In this study, we measured decorin expression immnohistochemically in $2 \mathrm{SCH}$ cases and examine feasibility of decorin stating for distinguishing between $\mathrm{SCH}$ and malignant vascular tumors.

\section{Case Report}

Case 1 was a 50-year-old man who first visited a general practitioner with a gradually enlarging subcutaneous tumor on his left sole that he had noticed at age of 47 year

\footnotetext{
*The authors declare no conflict of interest.

\#Corresponding author.
}

old. The practitioner took a small punch biopsy and consulted a pathologist. The pathology report stated that the tumor was composed of a proliferation of spindle cells and irregularly shaped vascular spaces, and suggested Kaposi's sarcoma. Thus, the general practitioner referred the patient to our clinic immediately. The plantar lesion was an 11-mm, firm tender subcutaneous tumor on the sole (Figure 1(a)). The surface of the tumor was slightly purplish and an operation scar was found on its center. His past history showed no apparent trauma or preceding inflammation. The cutaneous lesion was excised completely and there was no recurrence over 3 years.

Case 2 was a 16-year-old girl who presented with a 5year history of gradually enlarging subcutaneous tumors on her left sole that had recently become occasionally

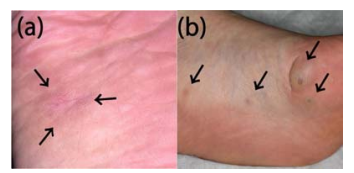

Figure 1. Clinical findings. Case 1: An 11-mm, firm tender subcutaneous tumor was found on the sole (a). Case 2: 16and 9-mm bluish subcutaneous tumors were seen on the thenar area of the left sole (b). Two similar 5-mm tumors were also found on the middle part of the sole. Arrows indicate location of tumors. 
painful. There was no family history of congenital abnormality and her past history was not contributory. Physical examination revealed a total of 4 subcutaneous masses separately on the left sole (Figure 1(b)). Relatively large blush tumors are 16- and 9-mm in size on the thenar area of the left sole. In addition, 2 small, 5-mm tumors were also found on the middle part of the sole, which the patient had not noticed before. The 16- and 9$\mathrm{mm}$ tumors were completely excised and there was no recurrence over 1.5 years.

Histologically, the tumors from both cases had 2 components: cavernous vascular spaces and cellular areas composed of spindle to epithelioid cells (Figures 2(a), (d)). The components' relative amounts differed in each lesion. A single layer of endothelial cells lined the vascular spaces. The spindle cells contained elongated, hyperchromatic nuclei and vacuolated cytoplasm, but did not exhibit atypia or mitotic figures (Figures 2(b) and (e)). The endothelial cells stained positively for CD31 and factor VIII-related antigen, whereas the spindle cells were negative. In addition, staining for CD34 was negative in the spindle cells. Thus, diagnosis of $\mathrm{SCH}$ was made based on the clinical and histological findings of both cases.

Next, we performed immunohistochemical analysis of serial sections using anti-decorin antibody (sc:73896, Santa Cruz Biotechnology, Santa Cruz, CA). The results demonstrated that immunoreactivity of decorin could be detected within the tumor mass. The expression within hemangioma was much stronger in the connective tissue stroma surrounding the blood vessels (Figures 2(c) and (f)).

\section{Discussion}

Angiogenesis involves remodeling of the extracellular matrix (ECM), proliferation, and migration of endothelial

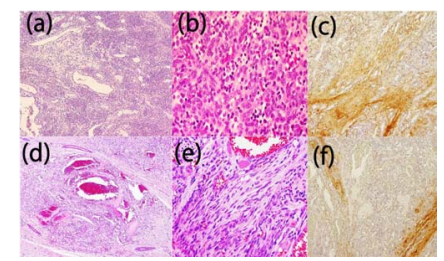

Figure 2. Histological and immunohistochemical findings. Histologically, the tumors from both cases had 2 components: cavernous vascular spaces and cellular areas composed of spindle to epithelioid cells (Case 1: (a), Case 2: (d)). A single layer of endothelial cells lined the vascular spaces. The spindle cells contained elongated, hyperchromatic nuclei and vacuolated cytoplasm but did not exhibit atypia or mitotic figures (Case 1: (b), Case 2: (e)). Immunohistochemical analysis using anti-decorin antibody demonstrated that immunoreactivity of decorin was detectable in the connective tissue stroma within tumor nests (Case 1: (c), Case 2: (f)). Magnification: (b), (f): 20×; (c), (g): 200×; (d), (h): 40×. cells [6]. A growing amount of evidence indicates that the multifunctional ECM molecule decorin, a member of the leucine-rich proteoglycan gene family, is associated with growth and differentiation processes. It is expressed in most collagen-rich mesenchymal tissues and up-regulated during angiogenesis of different conditions, especially in inflammatory processes [7]. In addition, decorin has been shown to regulate endothelial cell-matrix interactions during angiogenesis [5]. Furthermore, a current publication on benign and malignant vascular tumors demonstrated that expression patterns of decorin were differed from malignant to benign vascular tumors [4]. They showed that there was no detectable decorin immunoreactivity within the tumor mass in the Kaposi's sarcoma or angiosarcoma group. Instead, decorin was expressed in the connective tissue stroma lining the sarcoma tissue. In contrast to sarcomas, in benign hemangiomas, decorin immunoreactivity were observed also within the tumor mass, particularly in the connective tissue stroma surrounding the clusters of intratumoral blood vessels. They suggested that decorin might possess a suppressive effect on tumor angiogenesis and provide a useful biomarker to differentiate benign and malignant vascular tumors in patients [4].

$\mathrm{SCH}$ exhibits spindle cell proliferation and resembles Kaposi's sarcoma, which is a tumor of endothelial lineage whose spindle cells are susceptible to infection by human herpes virus 8 . Therefore, this study addresses decorin expression in the tissues of our $\mathrm{SCH}$ cases by immunohistochemical analysis using anti-decorin antibody. The results demonstrated that immunoreactivity of decorin was clearly detectable in the connective tissue stroma within tumor nests. This finding is compatible with the previous study that demonstrated negative and positive expressions in Kaposi's sarcoma and benign hemangioma, respectively [4]. Localization of decorin staining is supposed to be similar to collagen I. Combination of decorin and collagen 1 staining would give us more information concerning interpretation of malignant and benign vasucular tumors.

$\mathrm{SCH}$ was first perceived as sarcoma because of spindle cell proliferation, but this proliferation was subsequently found to be reactive. The presence of decorin expression in our cases also indicates that SCH have benign properties despite the spindle cell proliferation. Moreover, this study further increases the reliability of examination of decorin expression for differentiation between benign and malignant vascular tumors.

\section{REFERENCES}

[1] S. W. Weiss and F. M. Enzinger, "Spindle Cell Hemangioendothelioma: A Low Grade Angiosarcoma Resembling a Cavernous Hemangioma and Kaposi's Sarcoma," The American Journal of Surgical Pathology, Vol. 10, No. 
8, 1986, pp. 521-530. doi:10.1097/00000478-198608000-00001

[2] C. D. M. Fletcher, A. Beham and C. Schmid, "Spindle Cell Haemangioendothelioma: A Clinicopathological and Immunohistochemical Study Indicative of a Non-Neoplastic Lesion," Histopathology, Vol. 18, No. 4, 1991, pp. 291-301. doi:10.1111/j.1365-2559.1991.tb00849.x

[3] H. Järveläinen, P. Puolakkainen, S. Pakkanen, E. L. Brown, M. Höök, R. V. Iozzo, E. H. Sage and T. N. Wight, "A Role for Decorin in Cutaneous Wound Healing and Angiogenesis," Wound Repair and Regeneration, Vol. 14, No. 4, 2006, pp. 443-452. doi:10.1111/j.1743-6109.2006.00150.x

[4] H. H. Salomäki, A. O. Sainio, M. Söderström, S. Pakkanen, J. Laine, H. T. Järveläinen, et al., "Differential
Expression of Decorin by Human Malignant and Benign Vascular Tumors," Journal of Histochemistry and Cytochemistry, Vol. 56, No. 4, 2008, pp. 639-646.

[5] L. R. Fiedler and J. A. Eble, "Decorin Regulates Endothelial Cell-Matrix Interactions during Angiogenesis," Cell adhesion and migration, Vol. 3, No. 1, 2009, pp. 3-6.

[6] P. Carmeliet, "Angiogenesis in Health and Disease," $\mathrm{Na}$ ture Medicine, Vol. 9, No. 6, 2004, pp. 653-660. doi:10.1038/nm0603-653

[7] L. Nelimarkka, H. Salminen, T. Kupio, S. Nikkari, T. Ekfors, J. Laine, L. Pelliniemi and H. Järveläinen, "Decorin Is Produced by Capillary Endothelial Cells in Inflammation-Associated Angiogenesis," American Journal of Pathology, Vol. 158, No. 2, 2001, pp. 345-353. doi:10.1016/S0002-9440(10)63975-2 